\title{
Isolated angiitis of the CNS presenting as subarachnoid haemorrhage
}

\author{
Rajeev Kumar, Eelco F M Wijdicks, Robert D Brown Jr, Joseph E Parisi, \\ Charlotte A Hammond
}

\begin{abstract}
Isolated angiitis of the CNS (IACNS) commonly presents with recurrent ischaemic or haemorrhagic infarcts, but subarachnoid haemorrhage is rare. Three patients with IACNS and subarachnoid haemorrhage are reported. Florid granulomatous angiitis with Langhans and foreign body type giant cells was found at necropsy in a child with sudden death. In two other patients the diagnosis was made angiographically. In one patient multifocal infarcts on MRI became evident one week after subarachnoid haemorrhage despite initial treatment with prednisone. Subarachnoid haemorrhage may be the first presentation of IACNS. Characteristic radiographic findings may allow early diagnosis.
\end{abstract}

$(\mathcal{F}$ Neurol Neurosurg Psychiatry 1997;62:649-651)

Keywords: subarachnoid haemorrhage; vasculitis

Isolated angiitis of the CNS (IACNS) is a disorder of unknown aetiology and protean clinical manifestations. ${ }^{12}$ Presentation as an acute intraparenchymal haemorrhage has been increasingly reported ${ }^{3}$; however, subarachnoid haemorrhage as the sole defining feature has, to the best of our knowledge, been reported in only a few cases, ${ }^{1}$ only one of which was pathologically confirmed. ${ }^{4}$ We describe three new patients with distinctive clinical, radiographic, and pathological features.

\section{Neurology}

Department of

R Kumar

E F M Wijdicks

R D Brown Jr

Department of Atomic

Pathology

JE Parisi

Department of

Diagnostic Radiology, Mayo Clinic,

Rochester, MN, USA

C A Hammond

Correspondence to: Dr E F M Wijdicks, W8A Mayo Clinic, 200 First Street, SW, Rochester, MN 55905, USA.

Received 13 March 1996 and in final revised form 5 November 1996

Accepted 3 December 1996 treatment, but four hours after seeming otherwise well he was found dead.
Pathology

The formalin fixed brain weighed $1025 \mathrm{~g}$ and grossly displayed mild, diffuse cerebral oedema, evidenced by widened gyri and narrowed sulci. There was also mild, occasionally focal, duskiness of the cortical and deep grey matter, but normal subcortical and deep white matter. Pronounced subarachnoid haemorrhage was present, most prominently at the base, and the ventricular system contained a small amount of blood as well. An eccentric $3 \mathrm{~cm}$ non-aneurysmal dilatation of the distal left vertebral artery was present, but the vasculature was otherwise grossly normal. Microscopical examination showed florid granulomatous angiitis, characterised by a mixed inflammatory cell infiltrate with Langhans and foreign body type giant cells. These findings were most extensive and transmural at the vertebrobasilar junction, but were also present in small penetrating arterioles in the left hippocampus. Immunostaining for Varicella zoster virus was negative. The remainder of the necropsy (including examination of all other major cerebral and systemic arteries) disclosed only several small colonic ulcerations and no evidence of systemic vasculitis.

\section{PATIENT 2}

A healthy 50 year old woman had sudden onset of severe headache with associated nausea and vomiting. Neurological examination at admission was normal. Brain CT without contrast showed subtle hyperdensity over the convexity of the left hemisphere consistent with subarachnoid haemorrhage; the sylvian fissures and basal cisterns were clear (fig 1). Brain MRI confirmed these findings, but was otherwise normal. The CSF was bloody with greater than 10000 red blood cells $/ \mathrm{mm}^{3}$, protein $75 \mathrm{mg} / \mathrm{dl}$, normal glucose, and negative bacterial, fungal, viral, and mycobacterial cultures. Complete blood count was normal with the exception of a total leucocyte count of $16 \cdot 4$ $\times 10^{9} / 1$. The following additional studies gave normal results: coagulation profile, erythrocyte sedimentation rate, drug screen, urinalysis, ECG, antinuclear antibodies, and antibodies to extractable nuclear antigen. A four vessel cerebral angiogram performed less than 24 hours after onset of symptoms showed multiple areas of segmental alternating small artery 


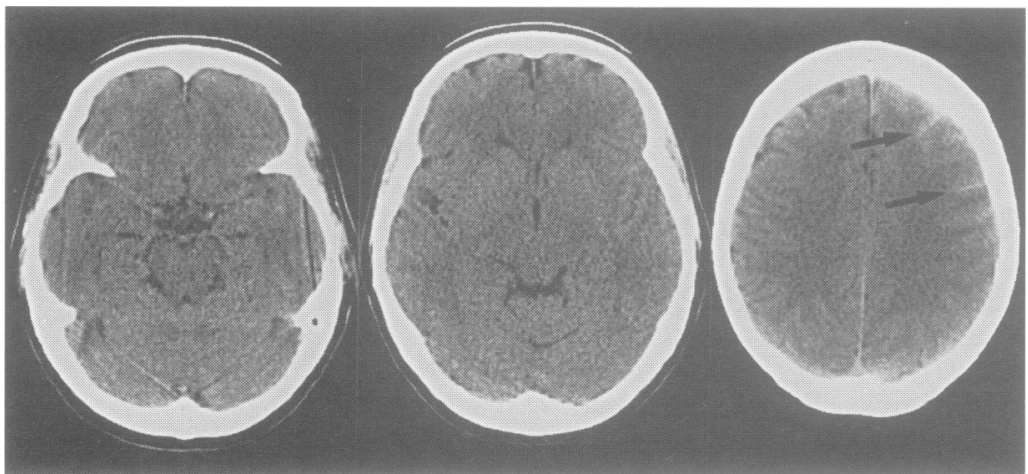

A

Figure 1 (A) CT showing subarachnoid haemorrhage at the convexity (arrow). No blood clots are identified in the basal cisterns. (B) Lateral view of cerebral angiogram; inset shows typical small artery

beading (arrows). (C) $M R I$ (flair sequence) showing right parietal haemorrhagic infarct and left frontal ischaemic infarct

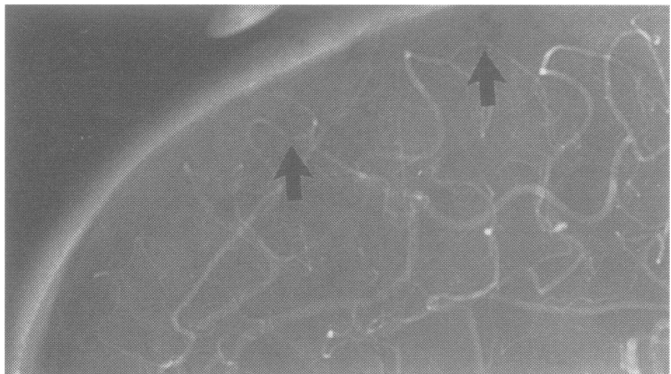

B

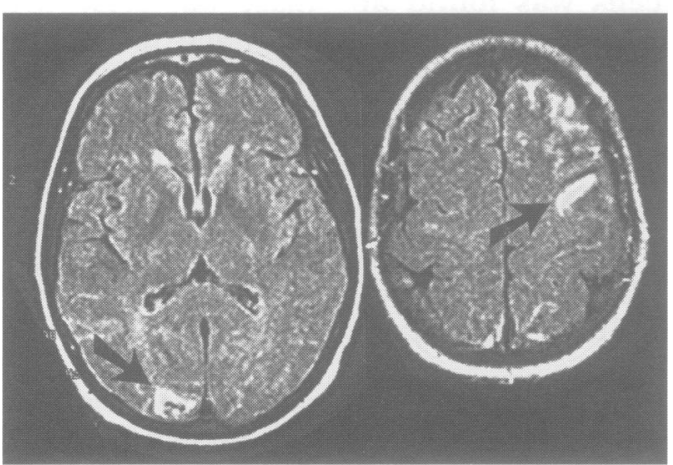

C

narrowing and dilatation ("beading") in bilateral anterior and middle cerebral artery distributions without evidence of an aneurysm or arteriovenous malformation (fig 1). Prednisone (60 mg daily) was given empirically for probable vasculitis and she was discharged in good clinical condition.

During the week after discharge, the patient experienced headaches and noted intermittent right hand and face numbness, difficulty reading and finding objects in front of her, and trouble using utensils with her right hand. When re-examined, she had fluctuating clinical findings of decreased right hand and face sensation and decreased rate of rapid alternating movements in her right hand. Brain MRI disclosed a new moderate sized left posterior frontal ischaemic infarct and a small right posterior parietal intraparenchymal haemorrhage (fig 1). Right frontal meningeal and cortical wedge biopsy was normal with the exception of one small area in the cortex which had a perivascular mononuclear cell infiltrate that was not diagnostic for vasculitis. Treatment with oral cyclophosphamide $(100 \mathrm{mg} /$ day) was begun and prednisone was continued. At one year follow up, she was asymptomatic and her neurological examination was normal. Cyclo-

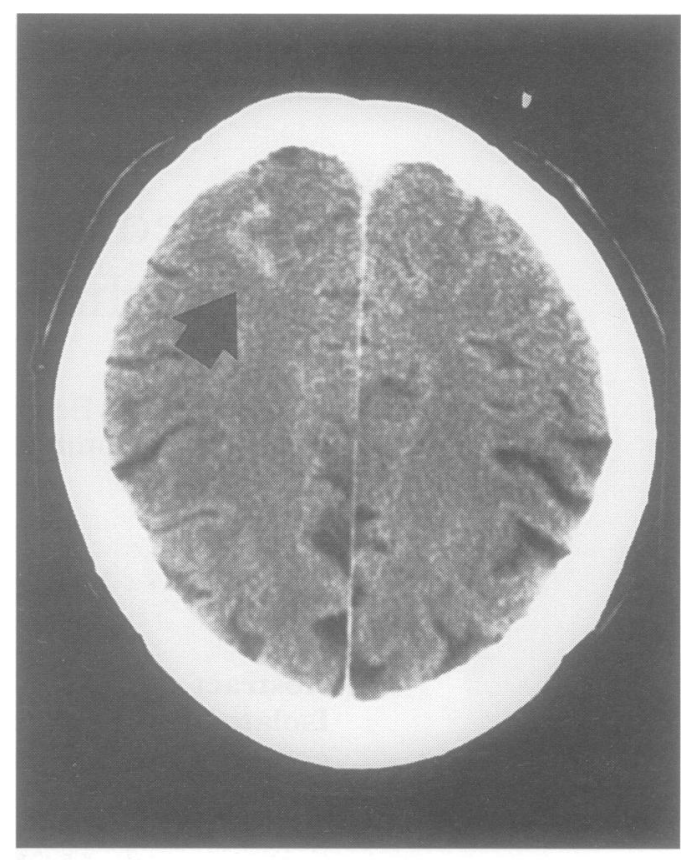

A

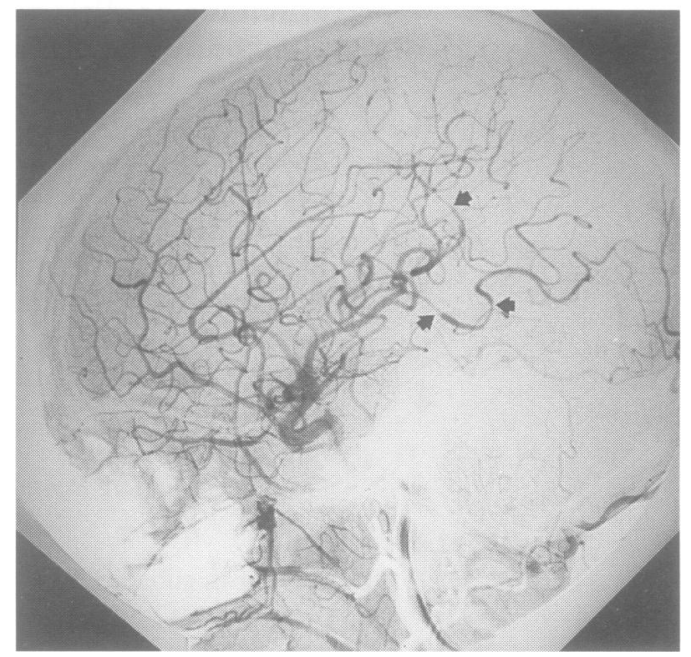

B

Figure $2 C T$ (two days after the ictus shows hyperdensity in the parietal lobe consistent with subarachnoid blood. Cerebral angiogram shows multiple areas of beading (arrows).

phosphamide and prednisone were tapered gradually over months and she remained asymptomatic 18 months after onset.

\section{PATIENT 3}

A healthy 53 year old woman with a history of hyperlipidaemia suddently developed an excruciating ("thunderclap") headache during a bowel movement. The headache persisted and temporarily worsened with Valsalva's manoeuvre. She denied any amphetamine use. Her neurological examination on admission was normal.

Brain CT (fig 2) showed subarachnoid blood over the left parietal convexity without blood in the basal cisterns or fissures. A cerebral angiogram showed multiple areas of arterial narrowing and dilatation (fig 2). Brain MRI confirmed the presence of subarachnoid blood but did not show cortical infarcts. Additional 
laboratory studies including CSF, antinuclear antibodies, hepatitis antibody panel, sedimentation rate, and drug screen were normal. Brain biopsy was refused.

She was treated with $60 \mathrm{mg}$ prednisone and had no neurological deficits or recurrence of the headache after three months follow up.

\section{Discussion}

Because IACNS has many well known clinical and angiographic mimickers, it is a difficult diagnosis and often one of exclusion. Our patients illustrate that IACNS may present with subarachnoid haemorrhage at virtually any age and with varying initial clinical severity. Before this report, only four cases of IACNS had been described in children. Matsell et al also reported diffuse pathological abnormalities; however, our paediatric case differs in its younger age of presentation (15 months $v$ six years), unique pathological features (large and small vessel granulomatous vasculitis $v$ small vessel involvement exclusively), and presentation as subarachnoid haemorrhage with sudden death. ${ }^{5}$ Shuangshoti reported subarachnoid haemorrhage secondary to IACNS in a 15 year old female with death resulting several days after the ictus. ${ }^{4}$ Vasculitic changes were present more diffusely in our case than in that reported by Shuangshoti, in which inflammatory changes were localised to the left posterior cerebral artery and adjacent small arteries only.

Our second and third cases are unique in their initial presentation. Only a small amount of peripherally located subarachnoid blood sparing the basal cisterns and sylvian fissures was found on neuroimaging. It may be argued that vasospasm could have produced the angiographic findings; however, vasospasm within 48 hours of ictus is extremely uncommon. Secondly, vasospasm induced by subarachnoid haemorrhage more commonly involves both large and small vessels. Instead, the angiogram correlates well with limited involvement of small cortically based arteries by vasculitis, one of which likely ruptured as a result of transmural inflammation with resultant vessel wall weakening. The development of multiple ischaemic and haemorrhagic infarcts soon after subarachnoid haemorrhage in one of our patients is consistent with vasculitis and not with delayed cerebral ischaemia. Unlike previously reported cases of IACNS presenting with subarachnoid haemorrhage, aneurysms were not identified by angiography and coexistent intraparenchymal haemorrhage was absent at presentation. Our report suggests that in patients with blood located at the convexity, IACNS should be considered in the differential diagnosis, which includes trauma, coagulopathies, and mycotic aneurysm. An essentially normal brain biopsy in our second case excludes well known mimicking disorders of IACNS, but does not exclude IACNS itself. ${ }^{2}$ This entity typically affects the cerebral vasculature in a patchy fashion, and pathological confirmation of the diagnosis may be found only at necropsy. ${ }^{6}$ The characteristic angiographic features, clinical worsening secondary to multifocal cerebral infarcts, and response to intensified immunosuppressive therapy supports our diagnosis of IACNS.

These cases show the need to recognise IACNS as a possible cause of subarachnoid haemorrhage at all ages. Location of subarachnoid blood at the convexity, lack of aneurysm or other identifiable cause of subarachnoid haemorrhage, and the presence of characteristic angiographic findings may hint at the diagnosis.

1 Cupps TR, Moore PM, Fauci AS. Isolated angiitis of the central nervous system: a prospective diagnostic and therapeutic experience. Am $\mathcal{F}$ Med 1983;74:97-105.

2 Moore PM. Diagnosis and management of isolated angiitis of the central nervous system. Neurology 1989;39:167-73.

3 Biller J, Loftus CM, Moore SA, et al. Isolated central nervous system angiitis first presenting as spontaneous vous system angiitis first presenting as spontaneou
intracranial hemorrhage. Neurosurgery 1987;20:310-5.

4 Shuangshoti S. Localized granulomatous (giant cell) angiitis of the brain with eosinophil infiltration and saccular aneurysm. F Med Assoc Thai 1979;62:281-9.

5 Matsell DG, Keene DL, Jimenez C, et al. Isolated angiitis of the central nervous system in childhood. Can $\mathcal{F}$ Neurol Sci 1990;17:151-4.

6 Hankey GJ. Isolated angiitis/angiopathy of the central nervous system. Cerebrovasc Dis 1991;1:2-15. 